\title{
Radiologists: threatened by a veritable identity crisis?
}

\section{Opinion}

Radiologists have always found it harder than representatives of other disciplines to ensure that their patients perceive and recognize them as true physicians rather than medical technicians. They are too strongly and too unilaterally associated with the machines they operate and with their products; in many cases, they are also too far removed from the pain and anxiety that prompted the patients to see a physician. Many radiologists have come to terms with this situation, accepting their role as representatives of a paramedical science more or less uncomplainingly and trying to make the best of things. No radiologist likes to be described as an academic photographer, but this unflattering epithet cannot be rejected entirely. The interpretation of conventional radiographs is an art and deserves to be recognized as such, but radiologists cannot claim to be the sole diagnostic agency and must share the prerogative of interpretation with others. Prime examples are specialty-specific diagnostic imaging e.g., for orthopedic specialists and the undisputed authority of other disciplines, such as dentistry, in adapting the imaging material to the case and treatment. The radiologist's status is inferior to the clinician's - in individual cases considerably so and radiologists do not diminish this inferiority when they ask their referring colleague what the latter expects to find from their report. Physicians who request examinations can supply most of the answers themselves, based on their specialist training and several years of practical experience in their discipline. The surgical speacialities also have the advantage that they can compare the images directly with the site. No consultative discussion or clinicalradiological meeting can compensate for this privilege.

\section{Diagnostic illustration freely available everywhere}

In the long era of analogue imaging, radiologists ' subservient role was confined to the interpretation of images. With the digital revolution, however, they are also suffering the same fate as their production, namely with data acquisition. Unlike the conventional radiograph, the digital radiograph is no longer a definitive, unique document, this product can no longer claim unrestricted copyright. Although radiologists obtain the dataset, they have no influence over what is done with it and what is made of it. The radiographic representation they have chosen is only one of many alternative ways of presenting the dataset optically. This limitation applies both to projectional radiography and diagnostic cross-sectional imaging. Whoever receives the raw data of an examination with the data pack can use the integrated program to postprocess the image impression on the computer and thus alter the content information at will. The computerized analyses and syntheses - computer-assisted diagnosis (CAD) included-do not just make the images potentially more presentable; they also make them more meaningful, easier, and more reliable to interpret. In assessing CT and MR images, knowledge of the subtleties of classical radiologic anatomy, has become dispensable. Two-dimensional, 3D and 4D reconstructions give the raw dataset an impressively sculpture-like quality; the computer-assisted evaluation at least partially anticipates the radiologist's diagnostic judgement, making it an alternative or competitor. The digital revolution has indivualized and boundlessly liberated data manipulation in diagnostic radiology. Anybody can be their own medical photographer, processing the dataset they have been given at will and emphasizing or toning

\author{
Volume 5 Issue 2 - 2017
}

\author{
Werner Albert Golder \\ Department of Radiology, France
}

Correspondence: Werner Albert Golder, Department of Radiology, France, Email werner.golder@orange.fr

Received: January 27, 2017| Published: February 08, 2017

down individual findings. Diagnostic illustration is thus gaining the potential to be as freely and ubiquitously available as photography has long since been in everyday life.

\section{New digital techniques for nonradiologists}

Compared with the technical options, such as those that can be used in reconstructing the dataset of CT colonography, the skill that radiologists have to demonstrate when they perform a conventional double-contrast examination of the large bowel seems like a poor craftsman's relic. Meanwhile, the image of the staff who show the dataset recipients how to configure the presentation and documentation has been enhanced. Therefore, the digital revolution has considerably boosted the prestige of the medical technical assistants, both in terms of patients and referring doctors. So far, teleradiology has had no such effect on the standing of the diagnostician. On the contrary, teleradiology is a further technical step on the way for radiologists to make themselves medically dispensable and to loose their prerogative of interpretation, even in emergency medicine. For telecommunication in radiology does not only mean medical activity remote from the patient; through the limitless dissemination of the image material, it also makes it possible for those who are not radiologists to be commissioned with the interpretation and/or to obtain a second or third opinion at the same time. Even in interventional radiology, the apparently new domain of the specialty of radiology, there has been a similarly critical development. In diagnostic radiology, at least nonspecialists only postprocess the datasets obtained, but the new digital techniques give nonradiologists the opportunity to make their own recordings within the framework of interventions. The hybrid operating room" makes this possible. Its array of equipmentangiography, CT, interventional MRI-practically invites the surgeons themselves to work in a minimally invasive fashion. During the preparation, operation, and control of image-guided interventions, they gain the necessary knowledge and skills to perform the interventions themselves later, more or less independently, and to integrate the data at the appropriate moment into open surgery. The digital imaging revolution has given a new dimension, both qualitative and quantitative, to competition in angiology, and also to other sectors in which minimally invasive work is done by both radiologists and the doctors giving clinical treatment who have the potential to refer. If this development continues at the same rate and to the extent outlined, 
the radiologist will soon become obsolete as the person responsible for configuring images, and he or she will be only one of many people who process the imaging datasets. Radiologists will have to come to terms with the fact that it will increasingly be possible for radiological assessments-unlike, say, reports from a pathologist or laboratory consultant-to be checked autonomously on the basis of the original data from the people who commissioned them, the patients and their relatives, and for them to be questioned and corrected thanks to postprocessing. Radiologists will have to accept that their chosen configuration of the images will therefore be only a provisional result in some cases and that this will devalue their craftmanship. And they will finally have to endure the fact that minimally invasive diagnosis and therapy are increasingly becoming an interdisciplinary specialty.

\section{Still the final authority in borderline cases}

This will not make diagnostic and interventional radiology a scientifically obsolescent model. Radiologists will still be the final authority in borderline cases, at least formally. Multidisciplinary statements and the unrivalled classification of variants at the borderline between normal and pathological will continue to earn them respect, and intelligent differential diagnosis will still be valued. But radiologists must learn to live with the paradox that although the digital imaging revolution and its immense configurative options are enlarging their methodological arsenal, this has not strengthened their authority. On the contrary, it is increasingly weakening it.

\section{Acknowledgements}

None.

\section{Conflict of interest}

The author declares no conflict of interest. 